(Aus dem chemischen Laboratorium des physiologischen Instituts zu Breslau.)

\title{
Zur Kenntniss \\ der Resorption einfacher, im besonderen stereoisomerer Zucker im Dünndarm.
}

Von

Junzo Nagano.

(Mit 1 Textfigur.)

Auf Grund seiner Versuche über die Resorption von Kochsalzlösungen und Blutserum war R. Heidenhain ${ }^{1}$ ) bekanntlich zu der Anschauung gelangt, dass zur Erklärung der Resorption im Dünndarm die Gesetze der Osmose nicht ausreichen, dass man vielmehr noch zur Annahme besonderer Triebkräfte, die ihren Sitz in den Zellen der Darmschleimhaut haben, gezwungen sei.

Seit den Arbeiten Heidenhain's sind eine Reihe von Untersuchungen erschienen, durch welche neue Gesichtspunkte für die Beurtheilung des "physikalischen Factors" der Resorption gewonnen worden sind. Es sei nur hingewiesen auf die Beobachtungen von Max Oker-Blom ${ }^{2}$, die zeigen, welche Bedeutung für das Verständniss des Resorptionsworganges die Vorstellungen haben, die wir uns von der Beschaffenheit der resorbirenden Membran machen, ferner auf die Arbeiten von R. Höbe $\mathrm{r}^{3}$ ), durch welche die Aufmerksamkeit auf die physikalisch-chemischen Eigenschaften der zu resorbirenden Lösung gelenkt wird. Er gibt an, dass die Geschwindigkeit, mit welcher versehiedene Salze im Dünndarm resorbirt werden, im Verhältniss ihrer Diffusionsgeschwindigkeiten steht.

Auch über die Resorption versehiedener Zucker stellte Höber eine Anzahl von Versuchen an. „Der Traubenzucker wird viel lang-

1) Dieses Archiv Bd. 56 S. 629. 1894.

2) Dieses Archiv Bd. 85 S. 543. 1901.

3) Dieses Archiv Bd. 74 S. 246. 1899. 
samer als Kochsalz, ziemlich erheblich schneller als Magnesiumsulfat resorbirt, die dem Traubenzucker isomere Galaktose ungefähr ebenso schnell wie der Traubenzucker und die Saccharobiosen Rohrzucker und Milchzucker langsamer als die beiden Monosen. ${ }^{\prime \prime}$

Für die Resorption der Zucker ist nach Höber die Diffusionsgeschwindigkeit ebenfalls von Bedeutung; denn der Traubenzucker, welcher langsamer als der Rohrzucker diffundirt, wird auch langsamer resorbirt. Auch die von ihm beobachtete geringere Resorptionsgeschwindigkeit des Traubenzuckers im Vergleich zum Kochsalz steht in Uebereinstimmung mit dieser Auffassung.

Daneben kommt aber, wie Höber ausführt, noch ein zweites Moment in Betracht, das Concentrationsgefälle. Je schneller der Zucker aus der Darmwand verschwindet, je grösser der Unterschied zwischen der Concentration der Lösung im Darm und dem Gehalt der Darmwand an resorbirter Substanz ist, um so schneller wird die Diffusion in die Darmwand hinein erfolgen.

Die Abfuhr des Zuckers aus der Darmwand kann durch verschiedene Umstände beeinflusst werden, unter Anderem durch die Leichtigkeit, mit der die Zellen der Darmschleimhaut den Zucker aufnehmen und verarbeiten.

Welche Rolle die Darmschleimhaut bei der Resorption der Zucker im Vergleich zu den Diffusionsgeschwindigkeiten spielt, lässt sich aber bisher nicht erkennen. Einen Anhalt zu ihrer Beurtheilung könnte man jedoch gewinnen, wenn man die Resorption verschiedener einfacher Zueker vergliche. Man darf wohl annehinen, dass die Diffusionsgeschwindigkeiten derselben keine grossen Unterschiede zeigen, weiss aber, dass ihr Verhalten gegenüber den Vorgängen in den lebenden Zellen sehr verschieden ist.

So wissen wir, dass die Zucker mit drei, sechs und neun Kohlenstoff-Atomen gärungsfähig sind, die mit fünf und sieben dagegen nicht, dass ferner die Pentosen weit schwieriger im Organismus verbrennen als die Hexosen. Innerhalb derselben Gruppe würden ferner die stereoisomeren Zucker zu prüfen sein, und zwar erstens diejenigen, bei denen die Gestalt des Moleküls sich verhält wie Bild und Spiegelbild, d. h. die d- und l-Reihen. Nur die ersteren sind gärungsfähig, und auch im Stoffwechsel der Thiere zeigen beide Reihen Unterschiede, wie sich aus dem Verhalten der Arabinosen ergibt, die im Laboratorium E. Salkowski's von C. Neuberg 
und J. Wohlgemuth ${ }^{1}$ ) untersucht wurden. Weiterhin wäre der Einfluss der anderen Configurationen festzustellen. Von den verschiedenen Hexosen z. B. ist es bekannt, dass ihre Assimilationsgrenze $\left.^{2}\right)$ im thierischen Organismus nicht die gleiche ist. Es wurde, um ein Beispiel anzuführen, nach Versuchen von G. Rosenfeld ${ }^{3}$ ) die Dextrose im Organismus völlig verbrannt, während bei Darreichung gleicher Mengen von Galaktose $16 \%$, von der Mannose $20 \%$ unverändert durch den Harn ausgeschieden wurden. Ein analoger Unterschied scheint auch bei den Pentosen zwischen Xylose und Arabinose zu bestehen.

Wie verhalten sich diese verschiedenen einfachen Zucker bei ihrer Resorption im Darm?

Der Ausfübrung einer solchen Untersuchung in vollem Umfange steht nur eine Schwierigkeit entgegen, nämlich die Beschaffenheit der Zucker, besonders auch in den erforderlichen Mengen.

Man muss sich vorläufig darauf beschränken, die Versuche mit den am leichtesten zugänglichen Zuckern anzustellen. Das sind von Aldohexosen die d-Glucose, die d-Galaktose und d-Mannose, von Ketosen die d-Fructose, von Aldopentosen die 1-Arabinose und 1-Xylose.

Bevor ich meine Versuche beschreibe, die ich auf Anregung und mit Unterstützung von Prof. F. Röhmann ausführte, will ich nicht zu erwähnen unterlassen, dass ausser von $\mathrm{H}$ ö ber auch von E. Héd on ${ }^{4}$ ) eine hierher gehörige Versuchsreihe veröffentlicht worden ist. Héd on füllte in Darmschlingen von Kaninchen gleiche Mengen einer mit dem Blutserum annähernd isotonischen Zuckerlösung und bestimmte nach zwei Stunden die Menge des im Darm noch vorhandenen Zuckers. Dabei fand er, dass Glucose und Galaktose besser als Arabinose und bei Weitem besser als Raffinose resorbirt wurden.

\section{Methode der Untersuchung.}

Die Resorptionsversuche im Darm können bekanntlich in zweierlei Weise angestellt werden. Man kann die zu untersuchende Flüssig-

1) Ber. d. deutseh. ehem. Ges. Bd. 34 S. 1745. 1901.

2) Vgl. F. Hofmeister, Arch. f. exp. Path. Bd. 25 S. 240.1889.

3) Centralbl. f. innere Med. $1900 \mathrm{Nr} .7$.

4) Compt. rend. de la Soc. de Biol. t. 52 p. 29, 41, 87. 1900. 
keit in abgebundene Darmschlingen hineinbringen und nach einer bestimmten Zeit den Darminhalt untersuchen; oder man stellt die Versuche an Hunden mit Vella'scher Darmfistel an. Beide Methoden haben ihre Vortheile, beide ihre Nachtheile ${ }^{1}$ ). Die erstere erfordert ein grosses Thiermaterial; die Narkose, die Eröffnung der Bauchhöhle, die Manipulationen mit der Schlinge können immerhin die Resorption in uncontrolirbarer Weise beeinflussen. Es erseheint mir ferner wegen der wechselnden Contraction des Darmes unmöglich, bei der Operation die Länge des Darmstückes, in welcher die Resorption vor sich gehen soll, mit der erforderlichen Genauigkeit festzustellen. Bei den Vell a-Fisteln kommt die Schlinge erst zur Benutzung, nachdem sie eine bestimmte Zeit ihrer normalen Function entzogen war. Die Darmschleimhaut der Sehlinge kann sich mit der Zeit verändern, besonders da sich meist früher oder später ein „katarrhalischer Zustand" der Schleimhaut einstellt, der sich in einer geringen Herabsetzung der Resorptionsfähigkeit und in einer Neigung zur Hypersecretion zu erkennen gibt. Die Gefahren, die hierdurch drohen, lassen sich aber vermeiden. Handelt es sich um den Vergleich von Lösungen, die an sich nicht reizend wirken, so braucht man nur die Reihenfolge der Versuche in entsprechender Weise zu wechseln, um vergleichbare Resultate zu erhalten. Der Vortheil, dass man an demselben Thiere ohne Narkose und stets an derselben Darmschlinge unter gleichen Bedingungen die Versuche anstellen kann, erschien so gross, dass zu den folgenden Versuchen nur Hunde mit Vellascher Darmfistel benuzt wurden.

Hund I, operirt am 22. April, getödtet am 29. Juli 1901. Nahtstelle des Darmes $230 \mathrm{~cm}$ vom Pylorus, $7 \mathrm{~cm}$ vom Coecum entfernt. Länge des ansgeschalteten Darmstückes $22 \mathrm{~cm}$. - Körpergewicht: Am 9. Mai 1901 $12,05 \mathrm{~kg}, 17$. Mai $13,5 \mathrm{~kg}, 12$. Juni $14 \mathrm{~kg}, 28$. Juli $15,3 \mathrm{~kg}$.

Hund II, operirt am 21. Mai, wegen Darmprolaps getödtet am 28. Juni 1901. Nahtstelle $20 \mathrm{~cm}$ vom Pylorus, $126 \mathrm{~cm}$ vom Coecum entfernt. Länge des ausgeschalteten Darmstückes $24 \mathrm{~cm}$. - Körpergewicht: Am 5. Juni $14,3 \mathrm{~kg}, 26$. Juni $15,3 \mathrm{~kg}$.

Hund III, operirt am 2. Juli, getödtet am 30. Juli 1901. Nahtstelle $63,5 \mathrm{~cm}$ vom Pylorus, $160 \mathrm{~cm}$ vom Coecum entfernt. Länge des ausgeschalteten Darmstückes $18 \mathrm{~cm}$. - Körpergewicht: Am 10. Juli 1901 12,2 kg, 28. Juli $12,0 \mathrm{~kg}$. 1895.

1) Vgl. W aymouth Reid. The Journ. of Physiology vol. 19 p. 240. 
Hund IV, Nahtstelle $50 \mathrm{~cm}$ vom Pylorus, $210 \mathrm{~cm}$ vom Coecum entfernt. Länge des herausgeschnittenen Darmstückes $27 \mathrm{~cm}$.

Bei allen Versuchen, die an diesen Hunden angestellt wurden, wurde in folgender Weise verfahren.

Die nach vorne gelegene Fistelöffnung wurde durch einen Gummiballon, der sich mit Wasser aufspritzen liess, verschlossen. In die hintere Fistelöffnung wurde eine Tamponcanüle eingeführt. Dieselbe bestand aus einem Metallrohr von $14 \mathrm{~cm}$ Länge und $4-5 \mathrm{~mm}$ lichtem Durchmesser; bei $a$ und $b$ waren zwei etwa $1 \mathrm{~mm}$ dicke und $5 \mathrm{~mm}$ breite mit Riefen versehene Streifen aufgelöthet, auf welche ein dünnes, cylindrisehes Stück Gummi (aus einem Handschuhfinger geschnitten) aufgebunden wurde. Durch $b$ geht ein dünnes Röhrchen, das an seinem Ende durch einen kleinen Hahn verschliessbar ist. An d fügt man mittelst eines Stückehens Gummischlauch eine mit Wasser gefüllte Spritze, treibt das Wasser zwischen Canüle und Gummiblase und schliesst den Hahn. Ueber das Ende $c$ der Canüle zieht man einen kurzen Gummischlauch, der durch einen Quetschhahn verschlossen werden kann. Man verbindet denselben durch ein Glasröhrchen mit einem längeren StückGummischlauch,

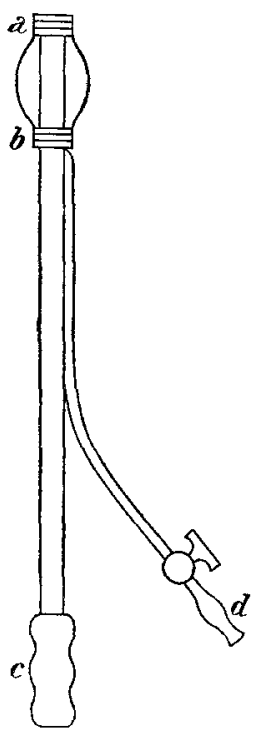
der zu einer Bürette führt, füllt Bürette und Schlauch mit der Flüssigkeit, welche resorbirt werden soll, und lässt bei demselben Hunde stets die gleiche Menge Flüssigkeit in die Fistel einfliessen. In der Canüle und dem Schlauch bleiben hierbei, gewissermaassen in einem todten Raum, 0,5-1 ccm. Der Verschluss ist ein vollkommener; einige Tropfen, welche während des Versuches von der Fistelöffnung herunterfallen, rühren von Darmsaft her, welcher von der nach aussen von den Verschlussstücken liegenden Schleimhaut unter dem Einfluss des von ihnen ausgeübten Druckes secernirt wird.

Der Hund stand während des ganzen Versuches ruhig in einem geeigneten Gestell. Jedesmal nach demselben wurde er, und zwar stets mit demselben Futter (200 g Fleisch und 1-2 Hundekuchen) gefüttert. Die Versuche sind also sämmtlich im nüchternen Zustande des Thieres angestellt. 
Nach einer bestimmten Zeit - im Allgemeinen nach einer Stunde - wurde die Flüssigkeit herausgelassen und gemessen. In allen Versuchen nach dem 7. Mai 1901 wurde zu Ende des Versuches die Fistel mit 0,5\%iger Kochsalzlösung ausgespült. In dem Ausgeflossenen wurde die Alkalescenz bestimmt, indem aus einer Bürette $1 / 10$ Normalschwefelsäure $\left(1 \mathrm{ccm}=4,0 \mathrm{mg} \mathrm{Na} \mathrm{CO}_{3}\right.$ ) bis zum Verschwinden der Blaufärbung von rothem Lackmoidpapier hinzugefügt wurde. Erhitzt man die so neutralisirte Flüssigkeit zum Sieden, so coagulirt das Eiweiss und lässt sich durch Filtration entfernen. Schneller und sicherer wird Letzteres erreicht, wenn man nach der Neutralisation eine bestimmte Menge von essigsaurem Natrium und Eisenchlorid zur Flüssigkeit hinzugefügt und dann zum Sieden erhitzt.

In der von Fiweiss befreiten Flüssigkeit wurde der Zucker durch Titriren mit Knapp'scher Lösung bestimmt. Der Titer wurde für jeden Zucker empirisch festgestellt.

\section{Versuche.}

\section{A. Resorption von Hexosen.}

1. d-Glucose.

Es kam reiner krystallisirter Traubenzucker von C. A.F. Kahlba um in Berlin zur Verwendung.

Versuche an Hund I.

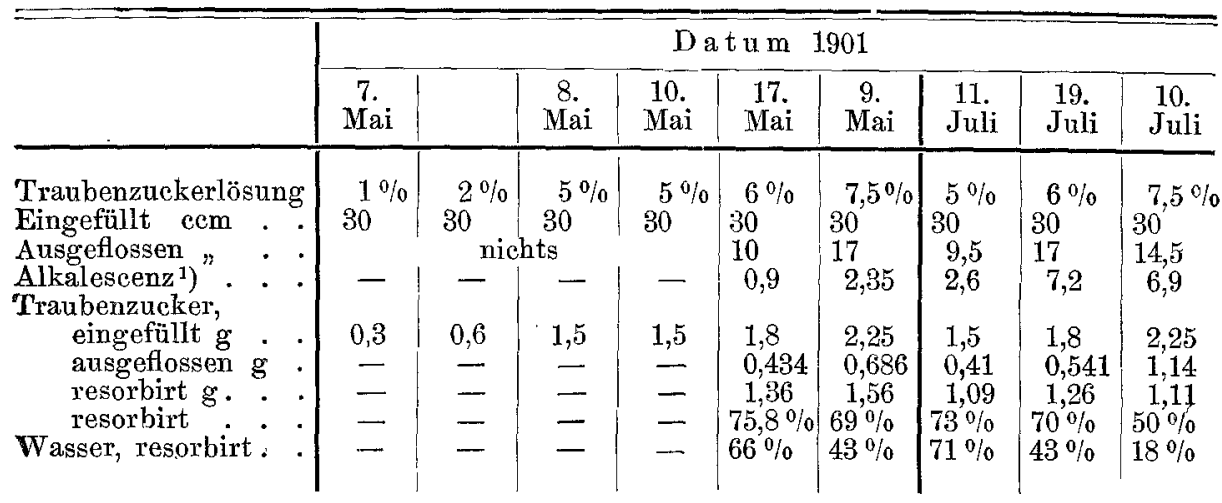

1) Alkalescenz bedeutet Cubikcentimeter $1 / 10$ Normal-Schwefelsäure. Bei der Berechnung der Wasserresorption wurde die Secretion von Darmsaft nicht berücksichtigt. 
Die Resorption des Traubenzuckers und Wassers war in den Versuchen aus dem Juli schlechter als in denen vom Mai; auch die Secretion von Alkali war wesentlich erhöht, beides ein Zeichen dafür, dass die Darmschlinge sich nicht mehr in einem völlig normalen Zustand befand.

Versuche an Hund III.

\begin{tabular}{|c|c|c|c|}
\hline & \multicolumn{3}{|c|}{ Datum 1901} \\
\hline & $\underset{20}{20}$ & $\begin{array}{c}22 . \\
\text { Juli }\end{array}$ & $\begin{array}{c}19 . \\
\text { Juli }\end{array}$ \\
\hline 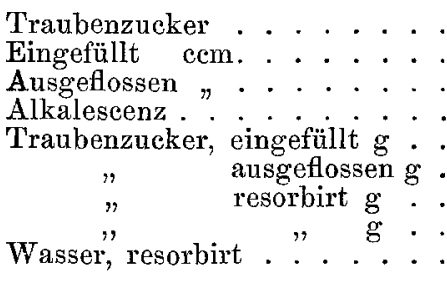 & $\begin{array}{l}5 \% \\
30 \\
14,5 \\
1,35 \\
1,5 \\
0,526 \\
0,974 \\
65 \% \\
51 \%\end{array}$ & $\begin{array}{l}5 \% \\
30 \\
15,0 \\
1,1 \\
1,5 \\
0,554 \\
0,946 \\
68 \% \\
50 \%\end{array}$ & $\begin{array}{l}6 \% \\
30 \\
18.0 \\
1,5 \\
1,8 \\
0,820 \\
0,980 \\
54,4 \% \\
40,0 \%\end{array}$ \\
\hline
\end{tabular}

In diesen an Hund III angestellten Versuchen wurde weniger Traubenzucker resorbirt als bei Hund I. Der Grund war, wie die Section ergab, nur der, dass die Darmschlinge kürzer war als die von Hund I, nämlich $18 \mathrm{~cm}$ gegenüber $22 \mathrm{~cm}$. Ein Vergleich der Resorption inı oberen und unteren Theile des Darmes ist auf Grund dieser Versuche nicht möglich. Beachtenswerth ist die geringe Alkalescenz im oberen Theile des Darmes, die auch nicht zunimmt, als mit steigender Concentration der Zuckerlösung die procentische Zuckerund Wasserresorption sinkt.

\section{2. d-Galaktose.}

Die Galaktose war aus Milchzucker durch Inversion mit Salzsäure gewonnen worden. Ihre Reinheit wurde. durch Bestimmung des Drehungsvermögens controlirt. Es betrug $[\alpha]_{20}^{D}+79,3^{\circ}$.

\section{Versuche an Hund $I$.}

Von einer 2 und $5 \%$ igen Galaktoselösung wurden $30 \mathrm{ccm}$ vollkommen resorbirt.

13. Mai 1901. Es wurden in die Darmschlinge eingefüllt $30 \mathrm{ccm}$ einer $7,5 \%$ igen Galaktoselösung. Es flossen aus $7 \mathrm{cem}$ mit einer Alkalescenz von 1,2. Das Ausgeflossene enthielt 0,37 $\mathrm{g}$ Galaktose. Resorbirt war $83,5 \%$ Galaktose und $76 \%$ Wasser. 
Versuche an Hund II.

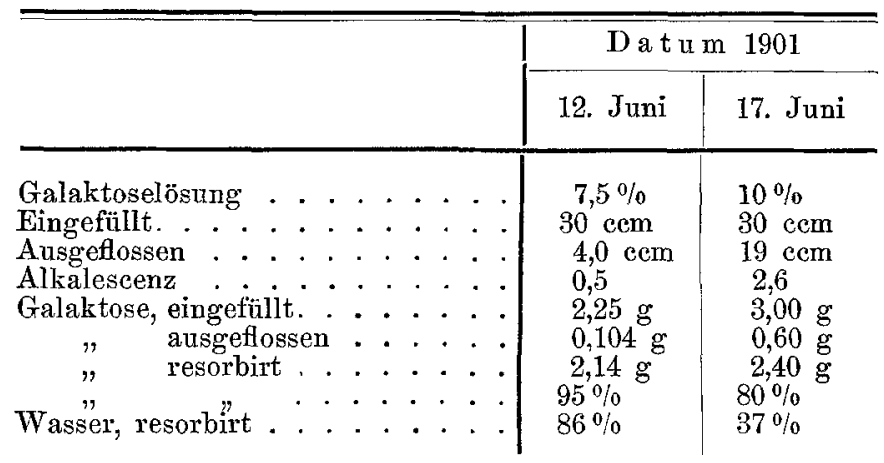

\section{Versuche an Hund III.}

24. Juli 1901. In die Darmschlinge wurden eingefüllt 30 ecm einer $7,5 \%$ igen Galaktoselösung, d. h. 2,25 g Galaktose.

Es flossen $24 \mathrm{ccm}$ aus mit einer Alkalescenz von 2,0. Das Ausgeflossene enthielt 1,12 g Galaktose. Resorbirt $1,13 \mathrm{~g}$, d. h. $50 \%$ Galaktose und $20 \%$ Wasser.

\section{3. d-Mannose.}

d-Mannose erhielten wir in Form eines weissen krystallinischen Präparates durch die Güte von Herrn Prof. Herzfeld, dem wir hierfür zu vielem Danke verpflichtet sind. Das Drehungsvermögen wurde in ca. $5 \%$ iger Lösung zu $[\alpha]_{20}^{D}=+13,6^{\circ}$ bestimmt.

Versuche an IInnd $\mathbf{I}$.

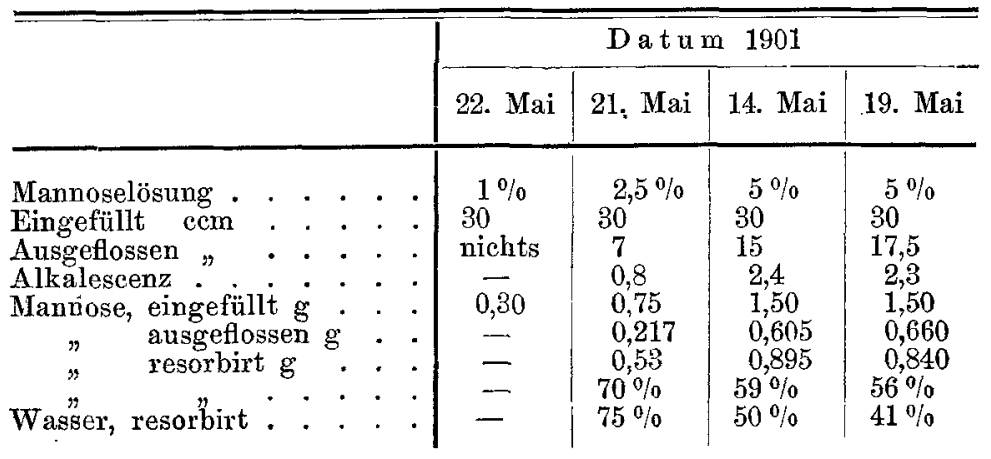

\section{4. d-Fructose.}

Zur Anwendung kam die krystallinische Laevulose von Kahlb a u m. Drehungsvermögen in c. $6 \%$ iger Lösung $[\alpha]_{20}^{D}-95,24^{\circ}$. 
Zur Kenntniss d. Resorption einfacher Zucker im Dünndarm.

Versuche an Hund I.

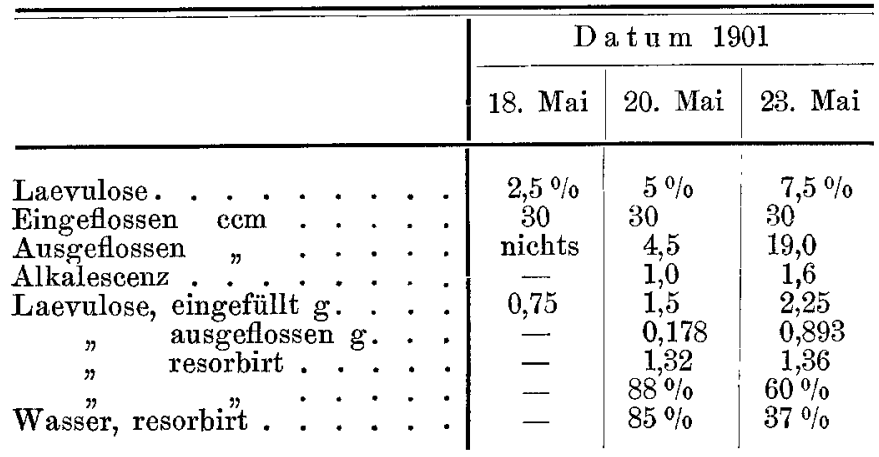

\section{B. Resorption von Pentosen.}

Die Versuche wurden angestellt mit l-Xylose und l-Arabinose. Erstere waren nach $\mathrm{Tollens}^{1}$ ) aus Weizenstroh, letztere nach Kiliani ${ }^{2}$ ) aus Kirschgummi dargestellt worden. Beide Präparate waren wiederholt aus Alkohol umkrystallisirt worden.

Versuche an Hund I.

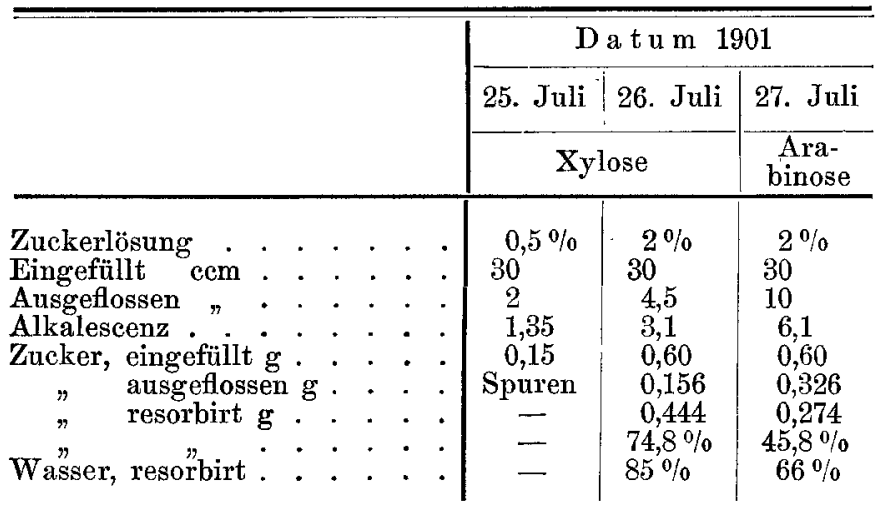

Versuche an Hund III.

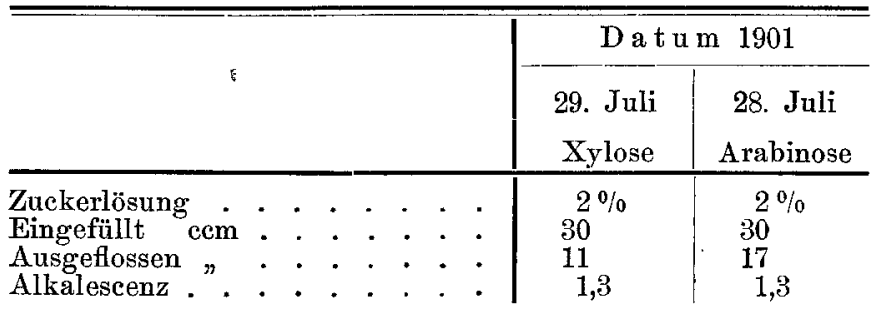

1) Annalen d. Chem. Bd. 271 S. 40.

2) Ber. d. deutsch. chem. Ges. Bd. 19 S. 3029. 
Versuche an Hund III. (Fortsetzung.)

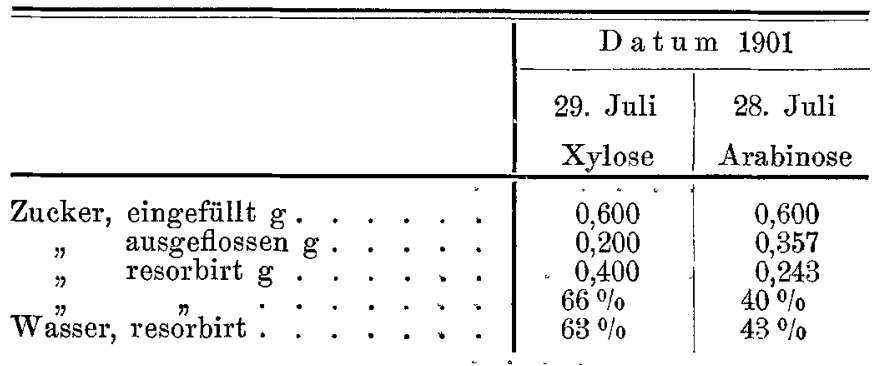

Ergebnissè.

Aus den mitgetheilten Versuchen lassen sich in Bezug auf die Geschwindigkeit, mit welcher die Lösungen einfacher Zucker im Darme aufgesaugt werden, die folgenden Resultate ableiten:

Die Resorptionsgeschwindigkeit stereoisomerer Zucker ist verschieden.

Das zeigt sich sowohl bei den Versuchen mit Hexosen wie mit Pentosen.

\section{Versuche an Hund $I$.}

d-Galaktose.

d-Mannose.

2-5\% \% Löge Lung vollkommen resorbirt $1 \%$ ige Lösung vollkommen resorbirt

$\begin{array}{cccccc}7,5 \% \text { ige } & 83 \% & 2,5 \% \text { oige } & & 71 \% & \\ & 5 \% \text { oige } & n & 56-59 \% & "\end{array}$

1-5\%ige Lösung vollkommen resorbirt 2,5\%ige Lösung vollkommen resorbirt

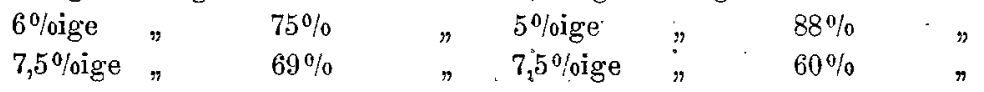

Wie man sieht, wird aus gleichconcentrirten, im vorliegenden Falle also äquimolekularen Lösungen die d-Galaktose etwas besser als die d-Glucose und diese sehr viel besser als die d-Mannose resorbirt.

Die Resorption der d-Fructose, welche sich als Ketose mit diesen drei Zuckern nicht auf eine Linie stellen lässt, ist etwas schlcchter als die der d-Glucose, aber besser als die der Mannose.

Bei den stereoisomeren Pentosen ist die Resorption der Xylose besser als die der Arabinose.

Von $2 \%$ I-Xylose wurden bei Hund I resorbirt $74,8 \%$, bei Hund II $66 \%$ "2\% l-Arabinose $", \quad " n \quad 45,8 \%, " n \quad, 40 \%$

Zugleich ergibt ein Vergleich der Pentosen und Hexosen, dass die Zucker mit fünf Kohlenstoff-Atomen langsamer 
Zur Kenntniss d. Resorption einfacher Zucker im Dünndarm. 399

als die mit sechs Kohlenstoff-Atomen im Darme aufgesaugt werden.

Die Geschwindigkeit der Wasserresorption zeigt bei der Resorption gleichconcentrirter Lösungen verschiedener Zucker ähnliche Unterschiede wie die Resorption des Zuckers.

\begin{tabular}{ccc} 
In den Darm eingefüllt & \multicolumn{2}{c}{ Resorbirt } \\
& $\%$ Zucker & Wasser \\
$7,5 \%$ Galaktose & 83 & $\%$ \\
$7,5 \%$ Glucose & 69 & 46 \\
$5 \%$ Glucose & \multicolumn{2}{c}{ vollkommen } \\
$5 \%$ Mannose & $56-59$ & $41-50$ \\
\hline $2 \%$ Xylose & 74,8 & 85 \\
$2 \%$ Arabinose & 45,8 & 66
\end{tabular}

Hiermit wäre im Wesentlichen, soweit dies durch die mitgetheilten Versuche möglich ist, die Eingangs gestellte Frage nach dem Verhalten verschiedener einfacher Zucker im Darm beantwortet.

Es zeigten sich aber dei diesen Versuchen noch einige Erscheinungen, die für das Verständniss der Darmresorption nicht ohne Interesse sind.

Bei demselben Zucker nimmt mit der Concentration der in den Darm eingefüllten Zuckerlösung die Resorption des W assers ab.

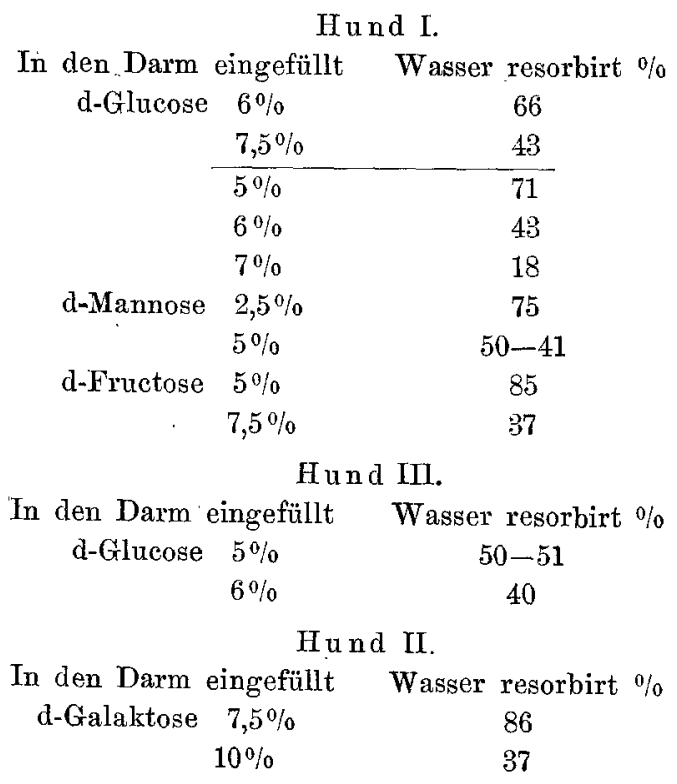


Die Erhöhung der osmotischen Spannung verzögert also den Austritt des Wassers aus dem Darm. Sie wirkt als wasseranziehende Kraft den Kräften, welche die Aufsaugung des Wassers herbeizuführen sich bestreben, entgegen.

Für den Zucker muss die Erhöhung der Concentration im Darm innerhalb gewisser Grenzen durch Erhöhung des Gefälles günstigere Bedingungen für die Resorption setzen. Ein solcher Einfluss ist auch vorhanden, wie die folgenden Zahlen zeigen.

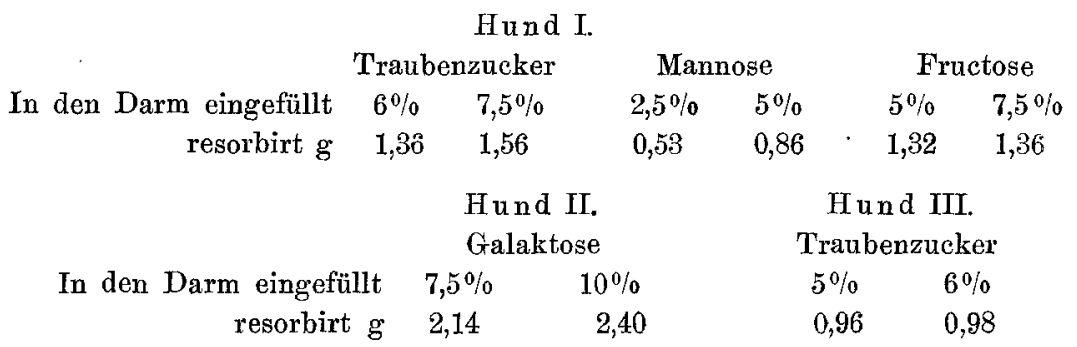

Um die Geschwindigkeit der Resorption des Wassers mit der des Zuckers zu vergleichen, kann man die Procentzahlen des resorbirten Zuckers durch die des Wassers dividiren; die für dieses Verhältniss gewonnenen Zahlen sind auf der folgenden Tabelle verzeichnet.

Verhältniss der Resorption von Zucker zu Wasser.

\begin{tabular}{|c|c|c|c|c|c|}
\hline \multicolumn{3}{|c|}{ Hund I. } & \multicolumn{3}{|c|}{ Hund II. } \\
\hline d-Galaktose & $7,5 \%$ & 1,1 & d-Galaktose & $10 \%$ & 2,16 \\
\hline \multirow[t]{2}{*}{ d-Glucose } & $7,5 \%$ & 1,6 & \multirow{2}{*}{\multicolumn{2}{|c|}{$7,5 \%$}} & 1,14 \\
\hline & $6 \%$ & 1,15 & & & \\
\hline \multirow[t]{2}{*}{ d-Fructose } & $7,5 \%$ & 1,62 & \multicolumn{3}{|c|}{ Hund III. } \\
\hline & $5 \%$ & 1,04 & d-Galaktose & $7,5 \%$ & 2,50 \\
\hline \multirow[t]{2}{*}{ d-Mannose } & $5 \%$ & 1,18 u. 1,36 & d-Glucose & $6 \%$ & 1,35 \\
\hline & $2 \%$ & 0,95 & & $5 \%$ & 1,26 u. 1,27 \\
\hline 1-Xylose & $2 \%$ & 0,88 & I-Xylose & $2 \%$ & 1,04 \\
\hline 1-Arabinose & $2 \%$ & 0,69 & 1-Arabinose & $2 \%$ & 0,93 \\
\hline
\end{tabular}

Dieselbe sagt aus, dass bei Hund I aus Zuckerlösungen, deren osmotische Spannung höher liegt als die des Blutes, d. h. höher als etwa $4,4 \%$, der Zucker schneller resorbirt wird als das Wasser; bei Lösungen von geringerer Spannung wird das Wasser schneller resorbirt als der Zucker. Bei Hund III sind die entsprechenden Quotienten etwas grösser. Hier nimmt der Zucker aus hypertonischen Lösungen bei derselben Concentration schneller ab als bei Hund I. 
Kinen anderen Ausdruck für dieselbe Erscheinung erhält man, wenn man aus den Zahlen der mitgetheilten Versuche die Zuckerconcentration berechnet, welche die im Darm enthaltene Lösung der "Resorptionsrückstand" am Ende des Resorptionsversuches hatte.

\begin{tabular}{clc}
\multicolumn{3}{c}{ Hund I. } \\
Resorbirte Lösung & Resorptionsrüekstand \\
Galaktose & $7,5 \%$ & $5,3 \%$ \\
Glucose & $6 \%$ & $4,3 \%$ \\
& $7,5 \%$ & $4,0 \%$ \\
& $5 \%$ & $4,3 \%$ \\
& $6 \%$ & $3,1 \%$ \\
Mannose & $7,5 \%$ & $4,1 \%$ \\
& $2,5 \%$ & $3,1 \%$ \\
& $5 \%$ & $4,0 \%$ \\
Fructose & $5 \%$ & $3,9 \%$ \\
& $5 \%$ & $3,9 \%$ \\
Xylose & $2 \%$ & $4,7 \%$ \\
Arabinose & $2 \%$ & $3,4 \%$ \\
\end{tabular}

Hund III.

\begin{tabular}{rcc}
\multicolumn{2}{c}{ Resorbirte Lösung } & Resorptionsrückstand \\
Galaktose & $7,5 \%$ & $4,2 \%$ \\
Glucose & $5 \%$ & $3,6 \%$ \\
& $5 \%$ & $3,7 \%$ \\
& $6 \%$ & $4,5 \%$ \\
Xylose & $2 \%$ & $1,8 \%$ \\
Arabinose & $2 \%$ & $2,1 \%$
\end{tabular}

Hund II.

$\begin{array}{cc}\text { Resorbirte Lösung } & \text { Resorptionsrückstand } \\ \text { Galaktose } \quad 10 \% & 3,2 \%\end{array}$

Füllt man in den Darm eine hypertonische Lösung, so sinkt die Concentration bei Hund I auf etwa $4 \%$, wenn man von der einen vielleicht nicht ganz richtigen Zahl von $3,1 \%$ absieht.

Die Concentration hypotonischer Lösungen nimmt dagegen zu. Die Concentration der 2,5\%igen Mannoselösung steigt auf 3,1\%, die der 2\%igen Xyloselösung auf $3,4 \%$, die der $2 \%$ igen Arabinoselösung auf $3,2 \%$.

Aehnliche Beobachtungen wurden für den Traubenzucker von O. Cohnheim ${ }^{1}$ ) und zum Theil auch von Hédon ${ }^{2}$ ) und Alber-
1) Zeitschr. f. Biol. Bd. 36 (18) S. 129.
2) a. a. 0 . 
ton $\mathbf{i}^{1}$ ) gemacht. Sie entsprechen den Resultaten, die Heidenhain und Gumilewski auch bei der Resorption von Kochsalzlösungen erhielten.

Vergleicht man die Versuehe an Hund I mit denen an Hund II und III, so findet man, dass bei Resorption derselben Zuckerlösung die noch nicht resorbirte Lösung im Darm der letzteren weniger Zucker enthält als bei ersterem. Die Lösungen von Glykose und Galaktose sinken bis $3,6 \%$, die $2 \%$ igen Lösungen von Arabinose und Xylose werden ohne Concentrationsänderung resorbirt, das heisst: im oberen Theile des Darmes wird der Zucker verhältnissmässig schneller resorbirt als das Wasser, im unteren das Wasser verhältnissmässig schneller als der Zucker.

Einige Bemerkungen seien auch über die Alkalescenz gemacht, welche die Zuckerlösungen einige Zeit, nachdem sie im Darm verweilt haben, annehmen.

Die Zahlen für dieselbe finden sich auf folgender Tabelle.

Alkalescenz.

\begin{tabular}{|c|c|c|c|c|c|c|c|c|}
\hline Lösung & $0,5 \%$ & $2 \%$ & $3 \%$ & $5 \%$ & $6 \%$ & $7,5 \%$ & $10 \%$ & \\
\hline \multirow[t]{3}{*}{ Glucose } & - & 一 & - & 一 & 0,9 & 2,35 & - & Hund I \\
\hline & - & 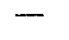 & - & $\left.[2,6]^{2}\right)$ & $\left.[7,2]^{2}\right)$ & $\left.[6,9]^{2}\right)$ & - & 3 \\
\hline & - & - & - & 1,35 & 1,5 & - & - & Hand 11 \\
\hline \multirow[t]{3}{*}{ Galaktose } & - & 一 & - & 1,1 & - & $\overline{1,2}$ & - & Hund I \\
\hline & - & $\ldots$ & - & - & - & 0,6 & 2,5 & Hund II \\
\hline & 一 & - & - & - & - & 2,0 & - & Hund III \\
\hline \multirow[t]{2}{*}{ Mannose . } & 一 & 0,8 & - & 2,4 & - & - & - & Hund I \\
\hline & - & - & - & 2,3 & - & $\overline{10}$ & 一 & $m$ \\
\hline \multirow{2}{*}{ Xylose } & $\overline{1,35}$ & $\overline{3,1}$ & - & 1,0 & - & 1,0 & - & $\eta$ \\
\hline & & 1,3 & - & - & - & - & & Hund III \\
\hline \multirow[t]{2}{*}{ Arabinose } & - & 6,1 & - & - & - & - & - & Hund I \\
\hline & & 1,3 & - & - & - & - & - & Hund III \\
\hline
\end{tabular}

Die Menge des Alkalis, welche bei der Resorption von 0,5 bis $10 \%$ igen Zuckerlösungen von $18-22 \mathrm{~cm}$ langen Darmschlingen abgesondert wurde, schwankte zwischen 4,5 und $35 \mathrm{mg} \mathrm{Na} \mathrm{CO}_{3}$.

1) Reale academia di Bologna 1901.

2) Die eingeklammerten Zahlen rühren von der bereits katarrhalischen Schlinge her. 
Bei der Resorption einer $5 \%$ igen Mannoselösung war sie grösser als bei der Resorption einer gleich concentrirten Lösung von Traubenzucker und Galaktose, noch stärker bei einer $2 \%$ igen Xylose oder gar Arabinoselösung.

Sie nahm bei demselben Zucker mit der Concentration der eingefüllten Lösung zu. (Vgl. d. fgd. Tab.)

\section{Versuche an Fund $\mathbf{I}$.}

\begin{tabular}{|c|c|c|}
\hline & $\begin{array}{l}\text { Von } 30 \text { cem Wasser } \\
\text { werden resorbirt }\end{array}$ & $\begin{array}{l}\text { Die Darmwand } \\
\text { secernirt Alkali }\end{array}$ \\
\hline $7,5 \%$ Galaktose & $76 \%$ & 1,2 \\
\hline $6 \%$ d-Glucose & $66 \%$ & 0,9 \\
\hline $7,5 \%$ & $43 \%$ & 2,35 \\
\hline 2,5\% d-Mannose & $75 \%$ & 0,8 \\
\hline $5 \%$ & 50 u. $41 \%$ & 2,4 u. 2,3 \\
\hline $5 \%$ d-Fructose & $85 \%$ & 1,0 \\
\hline $7,5 \%$ & $370_{10}$ & 1,6 \\
\hline $2 \%$ l-Xylose & $85 \%$ & 3,1 \\
\hline $2 \%$ l-Arabinose & $66 \%$ & 6,1 \\
\hline
\end{tabular}

Bei Beurtheilung der Ergebnisse, zu denen die mitgetheilten Versuche führen, muss zunächst noch einmal festgestellt werden, dass die Diffusionsgeschwindigkeiten der Zucker, mit denen die Versuche angestellt wurden, noch nicht bestimmt sind. Man ist daher auch nicht im Stande, zu sagen, ob die beobachteten Unterschiede in den Resorptionsgeschwindigkeiten der Zucker in einer Beziehung zu den Diffusionsgeschwindigkeiten stehen oder nicht.

Auf einen Unterschied im Gefälle könnte man es zurückführen, dass Pentosen langsamer resorbirt werden als Hexosen. Denn wie bereits erwähnt, werden diese auch langsamer und unvollkommener als jene im Organismus assimilirt. Auch die langsamere Resorption der Mannose lässt sich durch eine langsamere Assimilation erklären.

Anders steht es mit der Galaktose. Ihre Resorption ist gleich gut, ja anscheinend noch etwas besser als die der Glucose, ihre Assimilation aber erheblich schlechter. Will man an einer physikalischen Erklärung festhalten, so hat man daran zu denken, dass auch die Beschaffenheit der resorbirenden Membran für die Resorptionsgeschwindigkeit nicht gleichgültig sein kann. Es wäre immerhin möglich, dass diese für Zuckermoleküle einer bestimmten Gestalt 
404 Junzo Nagano: Zur Kenntniss der Resorption ete.

und Grösse (Galaktose) leichter durchgängig wäre als für andere (Glucose).

Wie dem auch sei, jedenfalls ist die Resorptionsgeschwindigkeit verschiedener einfacher, im besonderen auch die Resorptionsgeschwindigkeit von stereoisomeren Zuckern, d. h. von Zuckern, deren Molekül bei gleicher Zahl und Bindungsart der Atome eine verschiedene Gestalt hat, eine versebiedene. 